\title{
Memantine Protects Hippocampal Neuronal Function in Murine Human Immunodeficiency Virus Type 1 Encephalitis
}

\author{
Eric R. Anderson, ${ }^{1,2}$ Howard E. Gendelman, ${ }^{1,2,3}$ and Huangui Xiong ${ }^{1,2}$ \\ ${ }^{1}$ Center for Neurovirology and Neurodegenerative Disorders and Departments of 2 Pathology and Microbiology and ${ }^{3}$ Internal Medicine, University of \\ Nebraska Medical Center, Omaha, Nebraska 68198-5880
}

\begin{abstract}
Memantine, a low-to-moderate-affinity NMDA receptor antagonist, can be used to treat cognitive impairment associated with Alzheimer's disease. However, its potential neuroprotective effects for human immunodeficiency virus type 1-associated (HIV-1-associated) dementia are less well appreciated. To this end we studied hippocampal synaptic function in a severe combined immunodeficient (SCID) mouse model of HIV-1 encephalitis (HIVE). Human monocyte-derived macrophages (MDMs) infected with HIV-1 $1_{\mathrm{ADA}}$ were injected stereotactically into the caudate and putamen of SCID mice, generating HIVE. These brain subregions are among those most affected in humans. Impaired synaptic transmission and long-term potentiation (LTP) were detected in the CA1 region of hippocampal brain slices of HIVE mice. Memantine-treated HIVE mice showed significant improvements in synaptic function during frequency facilitation tests and LTP induced by high-frequency stimulation when compared with untreated animals. Immunocytochemical measures of neuronal antigens mirrored the neuronal physiological tests. These results demonstrate that memantine attenuates hippocampal synaptic impairment in murine HIVE and provide a rationale for its use in infected humans who experience cognitive decline.
\end{abstract}

Key words: memantine; HIV-1 encephalitis; monocyte-derived macrophages; neuroprotection; hippocampal slices; severe combined immunodeficient mice

\section{Introduction}

Adjunctive therapies for human immunodeficiency virus type 1-associated (HIV-1-associated) dementia (HAD) target indirect pathways of neuronal dysfunction (Lipton and Chen, 2004). Such pathways play a central role in neuronal destruction leading ultimately to cognitive, behavioral, and motor dysfunction (Kaul et al., 2001). Progressive HIV-1 infection in brain occurs commonly within mononuclear phagocytes (MPs; perivascular and parenchymal macrophages and microglia). Activation and viral infection lead to the secretion of a broad range of proinflammatory, chemotactic, viral, and metabolic factors collectively called "neurotoxins" (Zink et al., 1999; Kaul et al., 2001). For example, glial chemokines including, but not limited to, macrophage chemotactic factor enhance neural damage by directing MP ingress into the brain by providing increased numbers of viral host cells as sources of viral and cellular neurotoxins (Reinhart, 2003). Ultimately, widespread infiltration and activation of monocytederived perivascular brain macrophages incite brain inflammation, leading to paracrine and autocrine amplification of secretory products that affect neuronal injury and death (Lipton and Gendelman, 1995; Kaul et al., 2001).

Received May 18, 2004; revised July 2, 2004; accepted July 6, 2004.

This work was supported by National Institute of Neurological Disorders and Stroke Grants F31NS046233-01 (E.R.A. and H.X.), 3 R01 NS41862 (H.X.), P01 NS43985 (H.X. and H.E.G.), 2 R37 NS36126 (H.E.G.), and 2 R01 NS034239 (H.E.G.). We thank Drs. Larisa Poluektova and Huanyu Dou for assistance in the immunocytochemical assays and Drs. Mark Thomas and Jialin Zheng for careful review of this manuscript.

Correspondence should be addressed to Dr. Huangui Xiong, Center for Neurovirology and Neurodegenerative Disorders, University of Nebraska Medical Center, Omaha, NE 68198-5880. E-mail: hxiong@unmc.edu. DOI:10.1523/JNEUROSCI.1933-04.2004

Copyright $\odot 2004$ Society for Neuroscience $\quad$ 0270-6474/04/247194-05\$15.00/0
The linkage between diffusible MP neurotoxins and neuronal dysfunction is now a generally accepted mechanism for neuronal destruction for a number of neurodegenerative disorders (Ensoli et al., 2000; Jiang et al., 2001; Anderson et al., 2003). These may work through a single common pathway for neuronal injury that is operative via the NMDA receptor. NMDA receptors likely play a critical role in cognitive disease because they are an important component in the induction and maintenance of long-term potentiation (LTP), the physiological correlate of learning and memory. It was hypothesized that MP neurotoxic products, including glutamate and other excitotoxins, chronically may activate NMDA receptors, thereby leading to increased concentrations of intracellular calcium and, ultimately, to cell injury and death. Such pathways generally are believed to be a critical component of neurodegenerative processes that are operative during HIV disease (Doble, 1995; Sardar et al., 1999). Interestingly, MPassociated neurotoxicity is blocked by NMDA receptor antagonists in laboratory assays of HIV-1-associated neurotoxicity (Ushijima et al., 1995; Toggas et al., 1996; Kaul et al., 2001), implying that activation of NMDA receptors plays a role in HIV-1 neuropathogenesis.

Memantine is a well tolerated NMDA receptor antagonist that acts by blockade of the ion pore and has low affinity for the NMDA receptor, allowing it to disassociate readily from the channel (Parsons et al., 1995). Such kinetics allow it to block ongoing pathologic processes that involve chronic NMDA activation while simultaneously permitting normal neurophysiologic brain functions (Lipton and Chen, 2004). Memantine treatment has been directed primarily toward Alzheimer's disease and other senile dementias (Jain, 2000; Tariot et al., 2004). However, 
in vitro $\mathrm{HIV}-1$ proteins activate NMDA receptors, and this activation is ameliorated with memantine (Lipton, 1992; Nath et al., 2000). Furthermore, transgenic mice constitutively expressing HIV-1 glycoprotein 120 (gp120) benefit from memantine treatment (Toggas et al., 1996). To assess potential therapeutic modalities for HAD, we studied the effects of memantine on synaptic function in murine HIV-1 encephalitis (HIVE) by electrophysiological and immunohistochemical techniques.

\section{Materials and Methods}

Severe combined immunodeficient mouse model of HIVE and memantine administration. The severe combined immunodeficient (SCID) mouse model of HIVE was prepared as described previously (Anderson et al., 2003). Briefly, SCID mice (male C.B-17/IcrCrl-scidBR; 3-4 weeks old) purchased from Charles River Laboratories (Wilmington, MA) were inoculated into the caudate and/or putamen (striatum) with monocytederived macrophages (MDMs) infected with HIV $-1_{\mathrm{ADA}}$ (a macrophage tropic strain; HIVE) or uninfected MDMs (MDM) or were shamoperated (sham). Memantine $(5 \mathrm{mg} / \mathrm{kg}$ ) was administered daily by intraperitoneal injection beginning on the day of MDM implantation and continuing until the mice were killed $7 \mathrm{~d}$ later. The Institutional Animal Use and Care Committee (IACUC) of University of Nebraska Medical Center (IACUC \#94-126-08) approved all animal use procedures.

Preparation of hippocampal slices and electrophysiology. Transverse hippocampal slices were prepared from mice as previously described (Xiong et al., 1996). Briefly, mice were transported in microisolator cages to the electrophysiology laboratory within a biosafety level 3 (BSL-3) containment facility, anesthetized with Iso-Thesia, and decapitated, after which the brains were excised quickly. Hippocampi ipsilateral or contralateral to the injection site (caudate/putamen) were separated and placed in ice-cold $\left(4^{\circ} \mathrm{C}\right)$ oxygenated artificial CSF (ACSF) before sectioning. Previous studies performed by our laboratory have shown no difference in responses yielded from slices derived from the hippocampus ipsilateral and contralateral from the injection site (E. Anderson and H. Xiong unpublished observations); therefore, either hippocampus was used without preference. No hippocampi that were used showed any signs of needle trauma.

Transverse slices (400 $\mu \mathrm{m}$ thick) were cut from each dissected hippocampus with a tissue chopper. The slices were humidified in a custom holding chamber at room temperature for at least $1 \mathrm{hr}$ before being transferred into the recording chamber. In the recording chamber single hippocampal slices were submerged fully and perfused continuously with ACSF at a constant flow rate of $2 \mathrm{ml} / \mathrm{min}$ via a peristaltic pump (Rainin Instrument, Woburn, MA). The ACSF contained (in mM): 124 $\mathrm{NaCl}, 3 \mathrm{KCl}, 2 \mathrm{CaCl}_{2}, 2 \mathrm{MgCl}_{2}, 1 \mathrm{NaH}_{2} \mathrm{PO}_{3}, 26 \mathrm{NaCO}_{3}$, and 10 glucose. ACSF was equilibrated with $95 \% \mathrm{O}_{2} / 5 \% \mathrm{CO}_{2}$, giving a $\mathrm{pH}$ of $7.35-7.5$. The temperature of the perfusate was maintained at $30 \pm 1{ }^{\circ} \mathrm{C}$ with an automatic temperature controller (Warner Instrument, Hamden, CT). Field EPSPs (fEPSPs) were elicited by constant-current, low-frequency orthodromic stimulation $(0.05 \mathrm{~Hz})$ of Schaffer collateral/Schaffer collateral commissural axons with the use of an insulated (except for the tip) bipolar tungsten electrode. The stimulation intensity was adjusted to generate $\sim 40-50 \%$ of a maximal response. The evoked fEPSPs were recorded with an Axopatch-1D amplifier (Axon Instruments, Union City, CA) in the CA1 dendrite field (stratum radiatum). The recording microelectrodes were made from borosilicate glass capillaries with inner filaments that enabled quick backfilling. The tip diameter of the microelectrode was $\sim 5.0 \mu \mathrm{m}$ and had a resistance of $1-5 \mathrm{M} \Omega$ when filled with ACSF.

Frequency facilitation tests were performed at half-maximal fEPSP for each slice. After $20 \mathrm{~min}$ of slice acclimation, a stimulation burst consisting of 10 pulses was applied, and the resultant response was recorded. The slice was allowed to recover over the next $20 \mathrm{~min}$ before a different stimulation burst was given and recorded. The 10 pulse burst applied each time was at decreased interpulse intervals from 0.2 to $0.1 \mathrm{sec}$, respectively. The initial slope from each pulse in the recorded burst was analyzed and expressed as the percentage of the first pulse (taking the first pulse as 100\%). The recordings from the same experimental group at the same time point were averaged and graphed.

The ability of high-frequency stimulation (HFS; $100 \mathrm{~Hz}, 500 \mathrm{msec} \times$ 2) to induce LTP in the CA1 region of the hippocampus was examined. After a 30 min baseline recording, a HFS was delivered, and LTP was elicited. The initial slopes of the fEPSPs were measured and expressed as a percentage of baseline. Post-tetanic potentiation (PTP), short-term potentiation (STP), and LTP were determined at 1-2, 10-15, and 45-55 min after HFS, respectively.

Immunohistochemistry. Whole mouse brains were collected at necropsy at 3, 7, and $15 \mathrm{~d}$ after injection. Tissue was fixed in $4 \%$ paraformaldehyde for $48 \mathrm{hr}$ and embedded in paraffin. Serial coronal sections (5 $\mu \mathrm{m}$ ) were cut, and the dendritic arbor was detected with mouse antibodies against microtubule-associated protein-2 (MAP-2; Chemicon, Temecula, CA). Sections were viewed with a Nikon Eclipse (E800) microscope, and images were gathered by a MagnaFire digital camera and software (Optronics, Goleta, CA).

Statistical tests. All preceding studies were analyzed to determine significance by an ANOVA or two-tailed Student's $t$ tests. The level of significance was determined at $p<0.05$.

\section{Results}

We previously showed that synaptic dysfunction peaked in HIVE animals $7 \mathrm{~d}$ after MDM injection and that these deficits persisted for $15 \mathrm{~d}$ or longer (Zink et al., 2002; Anderson et al., 2003). To evaluate the neuroprotective effects of memantine, we conducted electrophysiological and immunohistochemical studies in the CA1 region of hippocampal brain slices prepared from HIVE, MDM, and sham mice. From each group one-half of the animals was administered memantine via intraperitoneal injection for $7 \mathrm{~d}$, and the other one-half served as untreated controls. At the time of death, hippocampal slices were dissected and tested by electrophysiological assays. Because of the inherent biohazards in working with HIV-1-infected animals and the technical difficulties in performing electrophysiological tests in a BSL-3 facility, the same numbers of experimental animals for each time point that was examined were not possible.

Synaptic transmission was assessed in the CA1 region after electrical stimulation of the Schaffer collateral commissural fibers at frequencies of 5.0 and $10.0 \mathrm{~Hz}$. These stimulations consisted of a 10 pulse burst with interpulse intervals of 0.2 and $0.1 \mathrm{sec}$, respectively. Synaptic strength evoked by electrical stimulation at $10.0 \mathrm{~Hz}$ was reduced significantly in slices taken from HIVE mice $(126.08 \pm 14.29 \%, n=6)$ in comparison with those from control animals (sham, $243.60 \pm 21.99 \%, n=5$; MDM, $242.19 \pm$ $21.95 \%, n=11)$. In contrast, HIVE mice treated with memantine exhibited a synaptic strength $(220.94 \pm 13.99 \%, n=6)$ similar to those recorded from controls (sham and MDM groups) (Fig. 1). In our previous study characterizing the synaptic deficits associated with HIVE, all input-output tests indicated no difference between the MDM and sham groups (Anderson et al., 2003). Therefore, the effects of memantine on MDM and sham were not tested in these input-output tests.

We next examined the effects of HIV-1 infection on activitydependent potentiation of synaptic efficiency in the hippocampus. The magnitudes of the three temporal components (PTP, STP and LTP) were decreased in HIVE animals in comparison to control animals (Table 1). The MDM animal group also exhibited diminished maintenance of LTP but to a lesser extent than that observed in the HIVE mice. In contrast, HIVE mice treated with memantine exhibited robust PTP, STP, and LTP responses that paralleled what were seen in sham-operated controls (Fig. 2). The memantine-treated MDM group exhibited higher LTP, but not PTP or STP, than the untreated MDM group. The sham 
group treated with memantine showed decreased PTP but similar STP and LTP when compared with the untreated animals.

MAP-2 immunostaining was used to assess whether memantine HIV-1 altered neuronal morphology as a consequence of HIV-1-infected MDMs. Notable differences in MAP-2 staining were visible in the Schaffer collateral pathways that harbor neuronal dendrites from CA1 pyramidal neurons in HIVE mice as compared with MDM or sham controls (Fig. 3). In contrast, the treatment of the HIVE group with memantine resulted in MAP-2 expression similar to that of controls. Importantly, the ability of memantine administration to spare hippocampal neurons from HIV-1-associated insults in HIVE mice supported the electrophysiological data. These differences also were easily discernible when the visible dendritic arbor in the HIVE mice treated with memantine was compared with that in untreated animals.

\section{Discussion}

The pathogenesis of neurodegenerative diseases commonly involves neurotoxic products produced by immunecompetent brain MP. During Alzheimer's and Parkinson's diseases, for example, brain MP activation and its inflammatory immune responses occur, resulting in chronic NMDA receptor activation and neuronal injury (Gray and Patel, 1995; Loopuijt and Schmidt, 1998; Mattson et al., 1999). Likewise, the murine model of HIVE used in this study demonstrates inflammatory neurotoxin activities originating from immune-competent brain MP (Persidsky and Gendelman, 2002). Injection of human HIV-1infected MDMs into the striatum of SCID mice causes disease in areas most affected in humans (Glass et al., 1993; Wiley and Achim, 1994; Persidsky et al., 1996). MDMs release soluble neurotoxins, and, after paracrine and autocrine amplification, such factors diffuse to affect regions distant from their primary locale. Because the activation of NMDA receptors can affect memory and learning (Latysheva and Rayevsky, 2003), we examined whether treatment with an NMDA antagonist such as memantine could improve synaptic transmission and plasticity and ameliorate subsequent neuropathology in the hippocampus, a brain region representative of learning and memory and apart from the initial source of the MP neurotoxins.

In vivo, memantine can reduce acute excitotoxic damage after exposure to glutamate and ameliorate behavioral deficits in animals infused with quinolinic acid (Keilhoff and Wolf, 1992; Misztal et al., 1996). Additionally, memantine can prolong LTP and as such improve memory retention (Seif el Nasr et al., 1990; Barnes et al., 1996; Zajaczkowski et al., 1997). Transgenic mice expressing HIV-1 gp120 exhibit an increase in hypothalamic-pituitaryadrenal axis activity that is dependent on NMDA receptor stimulation (Raber et al., 1996) and also benefit from memantinemediated neuroprotection (Toggas et al., 1996). Furthermore,
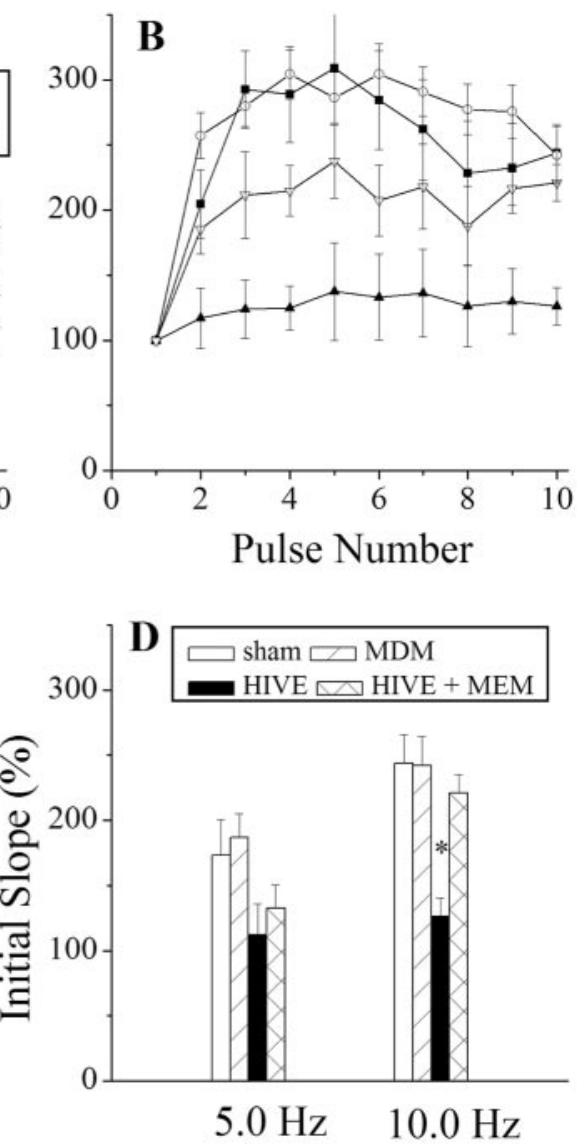

Figure 1. Evaluation of frequency facilitation in HIVE mice. The comparisons between frequency response recordings were frequency after MDM injection. Statistical comparisons were made with two-tailed Student's $t$ tests. Data points represent mean \pm SEM; asterisk denotes significant differences.

Table 1. Memantine restores HIV-1-associated inhibition of synaptic potentiation

\begin{tabular}{|c|c|c|c|}
\hline \multirow[b]{2}{*}{ Day 7} & \multicolumn{3}{|c|}{ Initial slope (\% basal level) } \\
\hline & PTP & STP & LTP \\
\hline Sham & $284.62 \pm 56.10$ & $334.97 \pm 48.227$ & $209.72 \pm 30.50$ \\
\hline Sham plus memantine & $205.73 \pm 14.47$ & $260.92 \pm 10.60$ & $229.52 \pm 18.69$ \\
\hline MDM & $279.79 \pm 60.29$ & $268.09 \pm 33.21$ & $160.32 \pm 14.007$ \\
\hline MDM plus memantine & $293.73 \pm 56.65$ & $262.43 \pm 24.07$ & $282.02 \pm 46.21]$ \\
\hline HIVE & $124.26 \pm 14.41]$ & $147.51 \pm 13.25]$ & $140.82 \pm 11.38]$ \\
\hline HIVE plus memantine & $476.28 \pm 76.66]$ & $305.07 \pm 26.01]$ & $263.38 \pm 42.02$ \\
\hline
\end{tabular}

Columns depict the resultant phases of synaptic potentiation (PTP, STP, and LTP) determined at 1-2, 5-10, and 45-55 min after high-frequency stimulation. Data are expressed as mean \pm SEM of the initial slope measured in percentage of basal activity. Significant differences were found between the HIVE group and controls in all phases by ANOVA $(p<0.005)$. Additionally, MDM exhibited significantly diminished LTP as well (sham, $n=8$; sham plus memantine, $n=5$; MDM, $n=9$; MDM plus memantine, $n=4$; HIVE, $n=8$; HIVE plus memantine, $n=5$; brackets indicate $p<0.05$; ANOVA; $t$ test).

cellular toxins secreted from HIV-1-infected MDMs can activate NMDA receptors aberrantly. These include quinolinic acid, glutamate, arachidonic acid and its metabolites, platelet-activating factor (Tsuzuki et al., 1989; Gelbard et al., 1994; Jiang et al., 2001; Arundine and Tymianski, 2003), and viral coat proteins (Lipton, 1992). With the administration of memantine to HIVE SCID mice, neuronal dysfunction resulting from chronic NMDA activation by diffusible toxins may be ameliorated.

Synaptic transmission and plasticity are important indicators of neuronal function. The frequency facilitation recordings ob- 

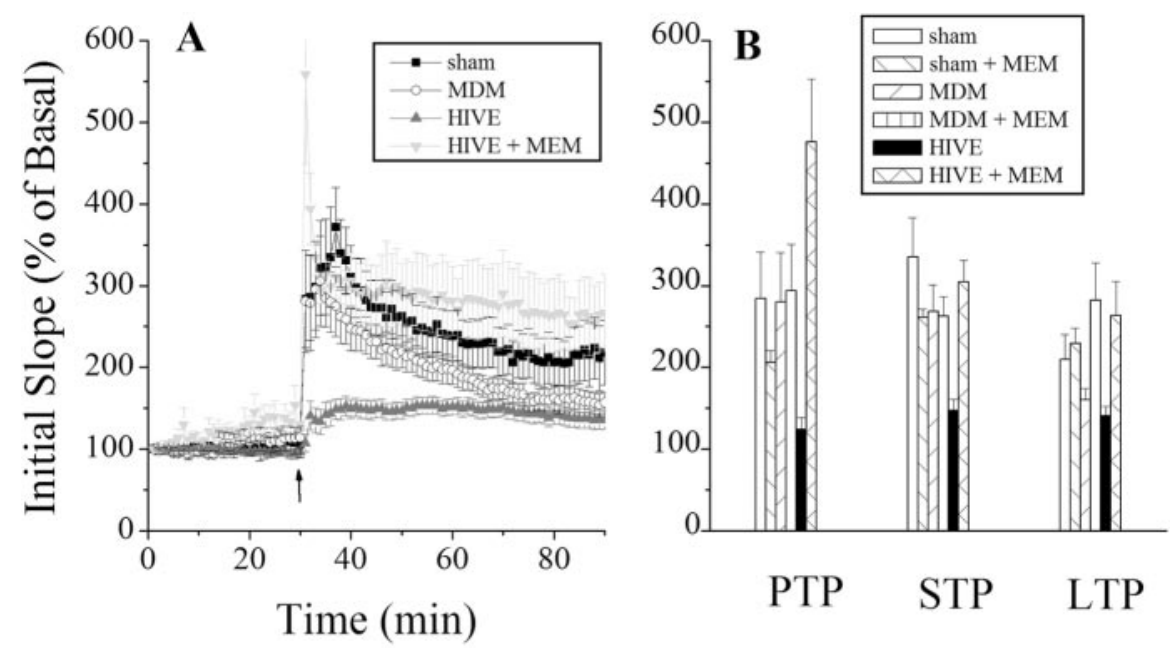

Figure 2. HIVE mice show marked reduction in LTP that is reversed after memantine treatment. $A$, Comparisons of synaptic potentiation induced in the hippocampi of SCID mice inoculated with HIV-1-infected MDMs inducing HIVE, uninfected MDMs (MDM), media alone (sham), and memantine-treated HIVE mice (HIVE + MEM). Baseline recordings were taken within the first $30 \mathrm{~min}$, followed by a burst stimulus resulting in LTP. Recordings were terminated $60 \mathrm{~min}$ after HFS. B, Distinct temporal components, which include PTP, STP, and LTP, are depicted via bar graphs. A significant reduction in PTP, STP, and LTP was observed in the HIVE group. The HIVE group treated with memantine exhibited a higher PTP, STP, and LTP than those components in the control groups. Data points represent mean \pm SEM; asterisk denotes significant differences.
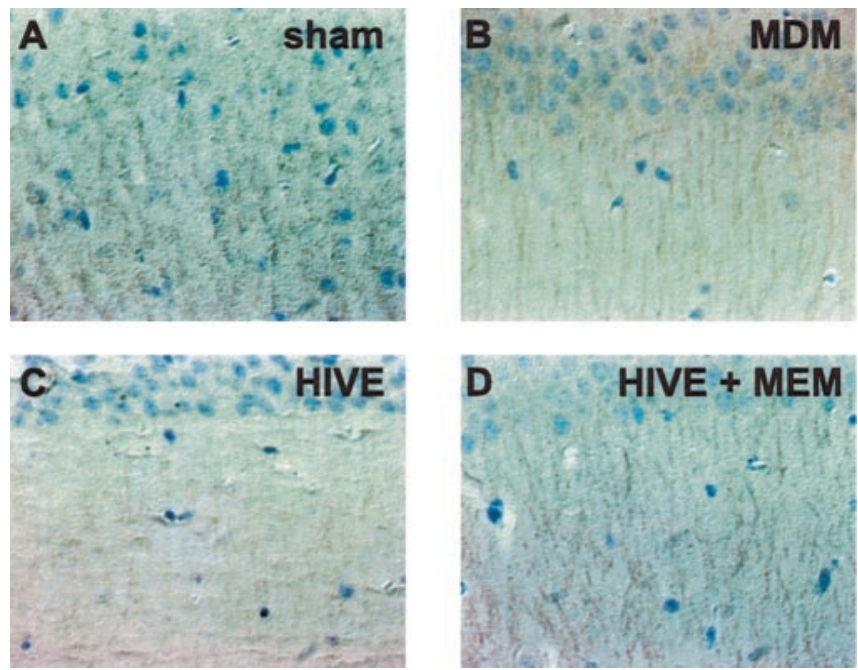

Figure 3. MAP-2 expression within the CA1 region of the hippocampus in HIVE mice treated with memantine. Mice from the four treatment groups (sham, MDM, HIVE, and HIVE + MEM; $A-D$, respectively) were killed $7 \mathrm{~d}$ after injection. All panels represent sections of SCID mouse brains immunostained with antibodies to MAP-2. Hippocampi were identified in coronal sections and then analyzed for MAP-2 immunoreactivity. The same region of the CA1 was selected in all samples. MAP-2 expression was reduced in the MDM and HIVE groups as compared with sham and HIVE + MEM. Primary antibodies were detected by Vectastain Elite kit, using DAB as a substrate, and tissue sections were counterstained with Mayer's hematoxylin, $60 \times$.

served in our murine HIVE model are indicative of neuronal dysfunction. However, in HIVE animals treated with memantine, synaptic dysfunction was not evident. Additionally, the LTP results from this study show greatly improved PTP, STP, and LTP in the HIVE group treated with memantine. The untreated HIVE group exhibited a severely diminished ability to evoke and maintain LTP in comparison to sham and MDM mice, as has been reported previously (Zink et al., 2002; Anderson et al., 2003). Furthermore, the MDM group treated with memantine also exhibited enhanced LTP in comparison with the untreated MDM group. Interestingly, we had reported previously that implanted
MDMs alone resulted in transient deficits in LTP maintenance at day 7, implying that transient MDM secretions occur after the xenograft (Zink et al., 2002; Anderson et al., 2003).

MAP-2 is associated closely with the NMDA receptor and normally is highly localized in the pyramidal cell dendrites comprising the CA1 region of the hippocampus (Buddle et al., 2003). Hence staining of normal CA1 dendritic neuropil for MAP-2 exhibits significant protein expression. Immunohistochemistry performed in this region demonstrated that MAP-2 antigen expression in HIVE mice was reduced significantly when compared with MDM and sham. In contrast, the memantine-treated HIVE group showed much higher expression of MAP-2, indicating protection of dendritic neuropil.

The mechanism underlying synaptic dysfunction in HIVE animals is not understood fully. Accumulating evidence indicates that overactivation of NMDA receptors by cellular and viral proteins plays a crucial role in $\mathrm{HIV}$-associated neural dysfunction. It is conceivable that chronic activation of NMDA receptors, and perhaps other membrane receptors in the HIVE mice, leads to the synaptic dysfunction and degeneration as observed in this study. Because both cellular and viral factors are present in the brains of HIVE animals, the protective effects of memantine on neuronal synaptic function suggest that the drug could affect positively a wide range of neurotoxic activities. In our previous work, we demonstrated that HIV-1 gp120 inhibited LTP in the CA1 region of rat hippocampal brain slices (Dong and Xiong, 2002). We also observed that preincubation of hippocampal slices with memantine significantly attenuated HIV-1 gp120-induced inhibition of LTP (J. Dong and H. Xiong, unpublished observations). This indicates that memantine could attenuate direct toxic effects of gp120 on neural cells. In keeping with these observations, findings from a recent phase II study of mementine for HIV-associated dementia suggest that memantine may improve neuronal metabolism in vivo by proton magnetic resonance spectroscopy and that it may prevent neurological progression in some patients at risk for worsening cognitive function (B. A. Navia, C. Yiannoutsos, and S. A. Lipton, personal communication). Together, these results suggest that memantine, when administered to HIVE mice, prevents neurodegenerative processes linked to chronic NMDA channel activation.

\section{References}

Anderson ER, Boyle J, Zink WE, Persidsky Y, Gendelman HE, Xiong H (2003) Hippocampal synaptic dysfunction in a murine model of human immunodeficiency virus type 1 encephalitis. Neuroscience 118:359-369.

Arundine M, Tymianski M (2003) Molecular mechanisms of calciumdependent neurodegeneration in excitotoxicity. Cell Calcium 34:325-337.

Barnes CA, Danysz W, Parsons CG (1996) Effects of the uncompetitive NMDA receptor antagonist memantine on hippocampal long-term potentiation, short-term exploratory modulation and spatial memory in awake, freely moving rats. Eur J Neurosci 8:565-571.

Buddle M, Eberhardt E, Ciminello LH, Levin T, Wing R, DiPasquale K, Raley- 
Susman KM (2003) Microtubule-associated protein 2 (MAP2) associates with the NMDA receptor and is spatially redistributed within rat hippocampal neurons after oxygen-glucose deprivation. Brain Res 978:38-50.

Doble A (1995) Excitatory amino acid receptors and neurodegeneration. Therapie 50:319-337.

Dong J, Xiong H (2002) Blockade of long-term potentiation by HIV-1 protein gp120 in rat hippocampal slices. Soc Neurosci Abstr 28:346.6.

Ensoli F, Fiorelli V, De Cristofaro M, Muratori DS, Novi A, Isgro A, Aiuti F (2000) Role of immune-derived diffusible mediators in AIDS-associated neurological disorders. Arch Immunol Ther Exp (Warsz) 48:259-266.

Gelbard H, Nottet H, Dzenko K, Jett M, Genis P, White R, Wang L, Choi Y-B, Zhang D, Lipton S, Swindells S, Epstein L, Gendelman H (1994) Platelet-activating factor: a candidate human immunodeficiency virus type-1 infection neurotoxin. J Virol 68:4628-4635.

Glass JD, Wesselingh SL, Selnes OA, McArthur JC (1993) Clinical-neuropathologic correlation in HIV-associated dementia. Neurology 43:2230-2237.

Gray CW, Patel AJ (1995) Neurodegeneration mediated by glutamate and beta-amyloid peptide: a comparison and possible interaction. Brain Res 691:169-179.

Jain KK (2000) Evaluation of memantine for neuroprotection in dementia. Expert Opin Investig Drugs 9:1397-1406.

Jiang Z, Piggee C, Heyes MP, Murphy C, Quearry B, Bauer M, Zheng J, Gendelman HE, Markey SP (2001) Glutamate is a mediator of neurotoxicity in secretions of activated HIV-1-infected macrophages. J Neuroimmunol 117:97-107.

Kaul M, Garden GA, Lipton SA (2001) Pathways to neuronal injury and apoptosis in HIV-associated dementia. Nature 410:988-994.

Keilhoff G, Wolf G (1992) Memantine prevents quinolinic acid-induced hippocampal damage. Eur J Pharmacol 219:451-454.

Latysheva NV, Rayevsky KS (2003) Chronic neonatal N-methyl-D-aspartate receptor blockade induces learning deficits and transient hypoactivity in young rats. Prog Neuropsychopharmacol Biol Psychiatry 27:787-794.

Lipton SA (1992) Memantine prevents HIV coat protein-induced neuronal injury in vitro. Neurology 42:1403-1405.

Lipton SA, Chen HS (2004) Paradigm shift in neuroprotective drug development: clinically tolerated NMDA receptor inhibition by memantine. Cell Death Differ 11:18-20.

Lipton SA, Gendelman HE (1995) Dementia associated with the acquired immunodeficiency syndrome. N Engl J Med 16:934-940.

Loopuijt LD, Schmidt WJ (1998) The role of NMDA receptors in the slow neuronal degeneration of Parkinson's disease. Amino Acids 14:17-23.

Mattson MP, Pedersen WA, Duan W, Culmsee C, Camandola S (1999) Cellular and molecular mechanisms underlying perturbed energy metabolism and neuronal degeneration in Alzheimer's and Parkinson's diseases. Ann NY Acad Sci 893:154-175.

Misztal M, Frankiewicz T, Parsons CG, Danysz W (1996) Learning deficits induced by chronic intraventricular infusion of quinolinic acid-protection by MK-801 and memantine. Eur J Pharmacol 296:1-8.

Nath A, Haughey NJ, Jones M, Anderson C, Bell JE, Geiger JD (2000) Synergistic neurotoxicity by human immunodeficiency virus proteins Tat and gp120: protection by memantine. Ann Neurol 47:186-194.

Parsons CG, Quack G, Bresink I, Baran L, Przegalinski E, Kostowski W,
Krzascik P, Hartmann S, Danysz W (1995) Comparison of the potency, kinetics, and voltage dependency of a series of uncompetitive NMDA receptor antagonists in vitro with anticonvulsive and motor impairment activity in vivo. Neuropharmacology 34:1239-1258.

Persidsky Y, Gendelman HE (2002) Murine models for human immunodeficiency virus type 1-associated dementia: the development of new treatment testing paradigms. J Neurovirol 8[Suppl 2]:49-52.

Persidsky Y, Limoges J, McComb R, Bock P, Baldwin T, Tyor W, Patil A, Nottet HS, Epstein L, Gelbard H, Flanagan E, Reinhard J, Pirruccello SJ, Gendelman HE (1996) Human immunodeficiency virus encephalitis in SCID mice. Am J Pathol 149:1027-1053.

Raber J, Toggas SM, Lee S, Bloom FE, Epstein CJ, Mucke L (1996) Central nervous system expression of HIV-1 gp120 activates the hypothalamicpituitary-adrenal axis: evidence for involvement of NMDA receptors and nitric oxide synthase. Virology 226:362-373.

Reinhart TA (2003) Chemokine induction by HIV-1: recruitment to the cause. Trends Immunol 24:351-353.

Sardar AM, Hutson PH, Reynolds GP (1999) Deficits of NMDA receptors and glutamate uptake sites in the frontal cortex in AIDS. NeuroReport 10:3513-3515.

Seif el Nasr M, Peruche B, Rossberg C, Mennel HD, Krieglstein J (1990) Neuroprotective effect of memantine demonstrated in vivo and in vitro. Eur J Pharmacol 185:19-24.

Tariot PN, Farlow MR, Grossberg GT, Graham SM, McDonald S, Gergel I (2004) Memantine treatment in patients with moderate to severe Alzheimer disease already receiving donepezil: a randomized controlled trial. JAMA 291:317-324.

Toggas SM, Masliah E, Mucke L (1996) Prevention of HIV-1 gp120induced neuronal damage in the central nervous system of transgenic mice by the NMDA receptor antagonist memantine. Brain Res 706:303-307.

Tsuzuki K, Iino M, Ozawa S (1989) Ion channels activated by quinolinic acid in cultured rat hippocampal neurons. Brain Res 481:258-264.

Ushijima H, Nishio O, Klocking R, Perovic S, Muller WE (1995) Exposure to gp120 of HIV-1 induces an increased release of arachidonic acid in rat primary neuronal cell culture followed by NMDA receptor-mediated neurotoxicity. Eur J Neurosci 7:1353-1359.

Wiley CA, Achim C (1994) Human immunodeficiency virus encephalitis is the pathological correlate of dementia in acquired immunodeficiency syndrome. Ann Neurol 36:673-676.

Xiong H, Baskys A, Wojtowicz JM (1996) Brain-derived peptides inhibits synaptic transmission via $\mathrm{GABA}_{\mathrm{B}}$ receptors in $\mathrm{CAl}$ area of rats. Brain Res 737:188-194

Zajaczkowski W, Frankiewicz T, Parsons CG, Danysz W (1997) Uncompetitive NMDA receptor antagonists attenuate NMDA-induced impairment of passive avoidance learning and LTP. Neuropharmacology 36:961-971.

Zink WE, Zheng J, Persidsky Y, Poluektova L, Gendelman HE (1999) The neuropathogenesis of HIV-1 infection. FEMS Immunol Med Microbiol 26:233-241.

Zink WE, Anderson E, Boyle J, Hock L, Rodriguez-Sierra J, Xiong H, Gendelman HE, Persidsky Y (2002) Impaired spatial cognition and synaptic potentiation in a murine model of human immunodeficiency virus type 1 encephalitis. J Neurosci 22:2096-2105. 